\title{
Annotations
}

\section{Magnetic resonance in diseases of the nervous system}

The anatomy and much of the pathology affecting the nervous system are well shown by the computer reconstructed sectional techniques of computed tomography and magnetic resonance imaging. The images from these techniques are quite similar and have common limitations of partial volume and reciprocation between contrast discrimination and spatial resolution, but the principles of their formation are radically different.

A computed tomogram is a sensitive measure of the linear absorption of $x$ rays at all points within a selected section of the child to be studied. It is achieved by measuring the intensity of an incident and emergent beam, traversing the slice from multiple angles. Where the bone around the neuraxis is a fairly even plate excellent images are achieved. Differential absorption of $x$ rays occurs, however, from the polychromatic beam where particularly dense bone surrounds the nervous system, and this is the basis of beam hardening artefacts, which detract considerably from the diagnostic value of computed tomography in the posterior and lower middle fossa, the spinal canal, and at craniocervical junction.

Certain nuclei behave like tiny bar magnets and when the body is placed in a strong static magnetic field they align along the force lines of the field and gyrate or precess around the axis of the field at a rate unique for type of nucleus, but within the radio frequency range and proportional to the strength of the field. By transmitting radio waves at the precession frequency the gyrating nuclei can be brought into phase and, at the same time, energy is added to the system. The synchronised nuclei emit a radio frequency signal that can be sufficiently strong to be recorded by a suitably placed receiving coil.

The most abundant nucleus in the body to behave in this way is the proton and it is the basis of all current magnetic resonance imaging. The superimposition on the static field of gradient fields allows precise localisation of the transmitting protons, their radio signals can therefore form the substrate of an image. Ràdio signals are free from the mechanical restraints which apply in computed tomography, so that magnetic resonance images can be obtained in any plane simply by modification of the direction of the gradient fields.
The image contrast is proportional to the strength of the signals at the time of imaging, which depends on:

(1) The density of the mobile protons in the tissue to be imaged. As cortical bone contains few mobile protons it produces neither signal nor image degradation, so that there are particular advantages in using magnetic resonance imaging as the primary mode of investigation in regions where beam hardening detracts from images produced on computed tomography.

(2) The capacity of the protons to retain the radio frequency energy. This is dependent on the physical state of the protons in the individual tissues. The energy is lost in two main ways. (a) By molecular collisions or thermal interactions. The time taken by the protons to magnetise in the strong magnetic field is a measure of this function. It is referred to as $T_{1}$ or spin lattice relaxation time. (b) By exchange of energy between the resonating protons. This causes dephasing at an exponential rate, referred to as $T_{2}$ or spin-spin relaxation time.

The importance of relaxation behaviour in formation of the image is evident from the fact that a $10 \%$ difference in tissue water concentrations can cause up to a $200 \%$ contrast difference, which accounts for the exquisite sensitivity of magnetic resonance imaging.

Other important factors influencing the image include:

(1) Mechanical movements of protons, as in blood flow and pulsation of the cerebrospinal fluid, between stimulation and recording of signal.

(2) Paramagnetic substances, which produce large local variations in the magnetic field; most typical examples are some of the naturally occurring iron compounds, including deoxyhaemoglobin, methaemoglobin, ferritin, and haemosiderin.

(3) Chemical shift. Protons in fat and water, being bound differently, resonate at slightly different frequencies so that images generated from them may not quite superimpose.

Judicious selection of sequence parameters for 
magnetic resonance can cause image brightness to reflect preferentially the influence of any one of these individual factors.

\section{Advantages and limitations of magnetic resonance imaging}

The lack of ionising radiation or, indeed, any other known biological hazard is of particular importance in young patients, especially if serial examinations are necessary. Sensitivity, freedom from bone induced artefact, and multiplanar imaging combine to make magnetic resonance imaging the optimum method of examining the nervous system. It has a few disadvantages in addition to the initial expense and limited accessibility. Even though it is now possible to record diagnostic data within seconds rather than the many minutes that used to be needed, ${ }^{1}$ heavy sedation or general anaesthesia is still necessary in about $15 \%$ of cases. Monitoring and life support apparatus compatible with the magnetic field and anaesthetic equipment free of ferromagnetic objects are available. Patients with electromagnetic or ferromagnetic implants, or metallic foreign bodies in sensitive situations, as near the eye, or aneurysms in or adjacent to the spinal cord or brainstem, cannot be placed within the magnet, whereas they can be examined by computed tomography.

\section{Contrast enhancement}

Gadolinium diethylenetriamine pentacetic acid (DTPA) shortens the $T_{1}$ relaxation time. With respect to the blood-brain barrier, it is similar to the iodine based contrast media used in computed tomography. ${ }^{2}$ Gadolinium enhancement has similar importance, but it is more sensitive. The sensitivity of magnetic resonance imaging reduces the necessity for a contrast agent in most cases, but it can be particularly useful in:

(1) The demonstration of (a) tumours strongly suspected clinically but invisible on unenhanced magnetic resonance imaging, such as small neuromas and metastases; (b) solid components within an extensive cystic or oedematous swelling, particularly in the spinal cord where exact localisation allows treatment or biopsy through a limited laminectomy ${ }^{3}$; and (c) selected cases of acute demyelination and small inflammatory foci and distinction from gliotic lesions.

(2) Reducing imaging time, particularly for volume acquisition.

No severe reactions to gadolinium DTPA have been recorded, and even minor hypersensitivity is very rare. ${ }^{4}$ Gadolinium is only available for routine clinical use in Germany, but licence applications have been filed in other countries, including Britian.

\section{Clinical applications}

\section{DEVELOPMENT AND MYELINATION}

Not only are all malformations and neuronal migration abnormalities at least as clearly shown by magnetic resonance imaging as by computed tomography, in addition, the extent of myelination and the effects of disease processes on its progress can be recognised at an early stage. For example, periventricular leucomalacia and haemorrhage from the germinal matrix in premature infants can be closely monitored and adverse effects of any complicating hydrocephalus on myelination can be finely judged, facilitating informed management.

\section{INFLAMMATORY LESIONS}

Lesions are usually visible in acute allergic encephalomyelitis, ${ }^{5}$ and infective encephalitis often shows non-specific changes on magnetic resonance imaging when computed tomography is negative: magnetic resonance should be the primary investigation when these diseases are suspected. In pyogenic and granulomatous inflammation, particularly in the supratentorial region, magnetic resonance imaging has little advantage over computed tomography; in granulomatous meningitis, enhancement in the basal cisterns is the important diagnostic feature and computed tomography is currently the modality of choice. Magnetic resonance shows plaques as regions of prolonged $T_{1}$ and $T_{2}$, most often in the deep cerebral white matter, in virtually all patients with established multiple sclerosis ${ }^{6}$; magnetic resonance imaging, though non-specific, may indicate the possibility of multiple sclerosis and repeat magnetic resonance imaging may show new plaques to support the diagnosis.

\section{LEUCODYSTROPHIES AND INHERITED ERRORS OF METABOLISM}

In leucodystrophies with abnormal computed tomography, magnetic resonance imaging reflects the distribution and often shows more extensive disease. Where family screening is to be undertaken, magnetic resonance is the procedure of choice. White matter abnormalities have been shown in two female carriers of Pelizaeus-Merzbacher disease, ${ }^{7}$ and the possibility of detecting subclinical changes in the carriers of other recessive or sex linked white matter diseases requires studying. In our limited experience, cases of metachromatic leucodystrophy that are negative on computed tomography have not shown abnormality on magnetic resonance imaging.

In inherited disorders of metabolism magnetic 
resonance imaging shows brain damage more exactly and at an earlier stage than computed tomography. The distribution may suggest particular groups of diseases, but the signal changes are rarely specific. The susceptibility of the basal ganglia to anoxia, metabolic acidosis, mitochondrial cytopathy, and Leigh's disease, Wilson's disease, HallovordenSpatz disease, and choreoacanthocytosis will suggest appropriate investigations for elucidation. Widened Virchow-Robin spaces, particularly in the peritrigonal regions, are common in the mucopolysaccharidoses, and infarcts and communicating hydrocephalus may complicate these diseases. Diffuse signal changes have been shown in the white matter in ceroid lipofuscinosis, in which computed tomography shows minor atrophy at most. Diffuse ischaemic changes or lacunar infarction, or both, are also shown at a relatively earlier stage in Anderson-Fabry disease.

\section{STROKE}

Ischaemia and infarction increase the water content of both grey and white matter, and produce high signal on $T_{2}$ weighted images, in concentrations many times less than detectable by computed tomography. The advantages of magnetic resonance imaging are accentuated in the posterior fossa; most brainstem infarcts are not shown by computed tomography.

The behaviour of haemorrhage on magnetic resonance imaging is complex. Acute haemorrhage, including subarachnoid haemorrhage, is well shown and easily interpreted on computed tomography. It will be evident on magnetic resonance imaging if reduction of $T_{2}$ is present due to the presence of deoxyhaemoglobin. Subacute and chronic haemorrhages are clearly and specifically shown by magnetic resonance imaging. ${ }^{8}$

\section{VASCULAR LESIONS}

The vascular anatomy outlined by the negative signal of flowing blood can show aneurysms and angiomatous malformations. Signal change reflecting intraluminal thrombus allows the diagnosis of arterial and dural sinus occlusion with appropriate sequences. ${ }^{9}$

\section{HEAD INJURY}

Extracerebral haematomas and the extent of parenchymal damage are better shown by magnetic resonance imaging than computed tomography. ${ }^{10}$ Nevertheless, computed tomography is easier to perform in the acute stages after injury and is currently the procedure of choice in identification of surgically significant intracranial haematomas. Magnetic resonance imaging may be particularly important in the assessment of residual brain damage.

\section{INTRACRANIAL TUMOURS}

The much greater sensitivity of magnetic resonance imaging, particularly in the posterior fossa and foramen magnum region, and the value of coronal and sagittal imaging in assessment of brain stem and juxtasellar regions applies to tumour detection and assessment. In optic glioma, for example, the lack of signal from the dense bone around the optic canal allows the full length of the optic nerve, in addition to the chiasm and proximal optic tracts, to be clearly shown. Multiplicity of lesions is more definitively established, and this may influence diagnostic possibilities in favour of metastases, lymphoma, or multifocal glioma. The distinction between tumour edge and oedema, of cystic from solid neoplasm, and of gliosis and fibrosis from neoplasia is no more accurate with magnetic resonance imaging than with computed tomography, and with similar contributions from contrast enhancement. The specificity of magnetic resonance imaging may be less than that of computed tomography in some cases, as, for example, in small craniopharyngiomas in which calcification may be a crucial diagnostic feature.

\section{SPINAL IMAGING}

The spinal cord and theca and the extrathecal segments of the nerve roots are well shown; the intrathecal nerve roots are more reliably shown on myelography or computed myelography.

All aspects of dysraphism-meningocoele, myelomeningocoele, lipomeningocoele, and diastematomyelia-are well shown. The site of the conus, cord tethering, the association of lipoma or dermoid, Chiari malformation or syringomyelia or other abnormality of the cord can all be determined. Arachnoid and neurenteric cysts expand the subarachnoid space and displace the neuraxis; their importance as compressing agents may be deduced from deformity induced on the cord. Ependymal cysts and dural ectasia may be recognised from the signal of their contents, being similar to that of cerebrospinal fluid.

Any cord swelling is evident, but distinction between cystic and solid intrinsic tumours, and even between tumours and acute demyelinating processes, may be impossible from consideration of the images alone. ${ }^{11-13}$

Cord compression and the nature of the compressing agent is usually demonstrated. The extent of extramedullary neoplasms or of stenosis of the spinal canal associated with bony dysplasias and metabolic diseases is shown and the dangers of spinal puncture are avoided.

Intramedullary angiomatous malformations are evident, but dural angiomas are not routinely shown. 
Magnetic resonance imaging is the favoured examination for elucidation of both acute and insidious spinal cord syndromes. Myelitis, in the absence of swelling, cannot be detected by any other study, and intrinsic cord damage from acute or chronic trauma is best shown by magnetic resonance imaging.

Computed tomography does have some advantages: fractures, dislocations, and osteophytes are more clearly shown; after penetrating trauma the possibility of retained metal close to the cord should be considered before placing the child in a magnet.

\section{Conclusion}

Magnetic resonance is the optimal method for primary diagnosis of structural disease of the brain and spinal cord, with the possible exception of acute haemorrhage and diseases where calcification is important for specific diagnosis. Until magnetic resonance imaging is freely available, however, computed tomography will remain as the screening investigation, followed by magnetic resonance imaging when it is doubtful or normal in cases in which structural disease is probable. In cases that are positive on computed tomography magnetic resonance imaging is likely to add important data only when multiplanar imaging is a critical consideration. Future prospects include the clinical introduction of sodium imaging, which may be useful in assessment of malignancy ${ }^{14}$ and in the management of oedema by assessment of osmolality. Phosphorous spectroscopy has not yet shown a specific abnormality in most pathologies, ${ }^{15}$ though a correlation with prognosis has been shown in perinatal ischaemia. Improvements in the resolution of the method and, in particular, the introduction of hydrogen spectroscopy, may produce more useful results, particularly in elucidation of metabolic abnormalities.

\footnotetext{
References

' Bydder GM, Payne JA, Collins AG, et al. Clinical use of rapid $\mathrm{T}_{2}$ weighted partial saturation sequences in MR imaging. $J$ Comput Assist Tomogr 1987;11:17-23.
}

2 Weinmann HJ, Laniado M, Mützel W. Pharmacokinetics of GdDTPA/dimeglumine after intravenous injection into healthy volunteers. Physiol Chem Phys Med NMR 1984;16:167-72.

${ }^{3}$ Bydder GM, Brown J, Niendorf HP, Young IR. Enhancement of cervical intraspinal tumors in magnetic resonance imaging with intravenous gadolinium-DTPA. J Comput Assist Tomogr 1985;9:847-51.

${ }^{4}$ Niendorf HP, Laniado M, Semmler W, Schörner W, Felix R. Dose administration of gadolinium-DTPA in MR imaging of intracranial tumors. AJNR 1987;8:803-15.

5 Atlas SW, Grossman RI. Goldberg HI, Hackney DB, Bilaniuk LT, Zimmerman RA. MR diagnosis of acute disseminated encephalomyelitis. J Comput Assist Tomogr 1986;10:789-801.

${ }^{6}$ Ormerod IEC, Miller DH, McDonald WI, et al. The role of NMR imaging in the assessment of multiple sclerosis and isolated neurological lesions: a quantitative study. Brain 1987; 110:1579-616.

${ }^{7}$ Bolthauser E, Schinzel A, Wichman W, Haler D, Valavanis A. Pelizaeus-Merzbacher disease: identification of heterozygotes with magnetic resonance imaging. Helv Paediatr Acta 1987;42: 337-9.

` Gomori JM, Grossman RI, Goldberg HI, Zimmerman RA, Bilaniuk LT. Intracranial haematomas: imaging by-high field MR. Radiology 1985;157:87-93.

9 Erdman WA, Weinreb JC, Cohen JM, Buja LM, Chaney M, Peshcock RM. Venous thrombosis: clinical and experimental MR imaging. Radiology 1986;161:233-8.

II Snow RB, Zimmerman RD, Gandy SE, Deck MDF. Comparison of magnetic resonance imaging and computed tomography in the evaluation of head injury. Neurosurgery 1986; 18: 45-52.

"Goy AMC, Pinto RS, Raghavendra BN, Epstein FJ, Kricheff II. Intramedullary spinal cord tumors: MR imaging, with emphasis on associated cysts. Radiology 1986;161:381-6.

12 Masaryk TJ, Modic MT, Geisinger MA, et al. Cervical myelopathy: a comparison of magnetic resonance and myelography. J Comput Assist Tomogr 1986;10:184-94.

${ }^{13}$ Rubin JM, Aisen AM, DiPietro MA. Ambiguities in MR imaging of tumoral cysts in the spinal cord. J Comput Assist Tomogr 1986;10:395-8.

14 Perman WH, Turski PA, Houston LW, Glover GH, Hayes CE. Methodology of in vivo human sodium MR imaging at $1.5 \mathrm{~T}$. Radiology 1968;160:811-20.

15 Turski PA, Perman WH, Hald JK, Houston LW, Strother CM, Sackett JF. Clinical and experimental vasogenic oedema: in vivo sodium MR imaging. Radiology 1986;160:821-5. 